\title{
ERGODIC MAXIMUM PRINCIPLE FOR STOCHASTIC SYSTEMS
}

\author{
CARLO ORRIERI, GIANMARIO TESSITORE, AND PETR VEVERKA
}

\begin{abstract}
We present a version of the stochastic maximum principle (SMP) for ergodic control problems. In particular we give necessary (and sufficient) conditions for optimality for controlled dissipative systems in finite dimensions. The strategy we employ is mainly built on duality techniques. We are able to construct a dual process for all positive times via the analysis of a suitable class of perturbed linearized forward equations. We show that such a process is the unique bounded solution to a Backward SDE on infinite horizon from which we can write a version of the SMP.
\end{abstract}

\section{INTRODUCTION}

We consider an optimal control problem with the following controlled dissipative stochastic state equation

$$
\left\{\begin{array}{l}
d X_{t}=b\left(X_{t}, u_{t}\right) d t+\sigma\left(X_{t}, u_{t}\right) d W_{t}, \quad t \geq 0 \\
X_{0}=x
\end{array}\right.
$$

and an ergodic cost functional (e.g. a functional that depends only on the asymptotic behaviour of the state and of the control) such as:

$$
\begin{gathered}
J^{\inf }(u(\cdot))=\liminf _{T \rightarrow \infty} \frac{1}{T} \mathbb{E} \int_{0}^{T} f\left(X_{t}, u_{t}\right) d t, \\
J^{\sup }(u(\cdot))=\limsup _{T \rightarrow \infty} \frac{1}{T} \mathbb{E} \int_{0}^{T} f\left(X_{t}, u_{t}\right) d t .
\end{gathered}
$$

In the above the state $X$ is a $\mathbb{R}^{n}$-valued process and $\left(W_{t}\right)_{t \geq 0}$ is a $d$-dimensional Wiener process. Moreover the drift $b$ and diffusion $\sigma$ satisfy a joint monotonicity condition. Finally the control process $\left(u_{t}\right)$ is progressively measurable and takes values in a non-empty convex subset $U \subset \mathbb{R}^{l}$.

We refer to this setting as ergodic control problem. The choice of the functionals refers to "minmin" and "minmax" formulation. Our aim is to find a correct formulation of the stochastic maximum principle (SMP) in the sense of Pontryagin, by means of which we have at our disposal some necessary (and sufficient) condition for optimality.

Alternatively, under stronger regularity assumptions, one can use the dynamic programming and derive the Hamilton-Jacobi-Bellman equation whose solution gives the optimal cost and the optimal feedback control. In finite dimensions, the first result was obtained in the paper by Mandl [13], later generalized by Bokar and Gosh in [3]. For further generalizations of such an HJB approach, both in finite and infinite dimensional framework both by analytic and by probabilistic tools see e.g. [8, 7, 11, 6, 17, 2, 1] We also refer to [9] for a survey on recent results obtained in this direction.

Nevertheless, it is by now well known that, even if it only provides necessary ( only under strong convexity requirements also sufficient) optimality conditions, the SMP normally requires much less regularity and structural condition allowing for instance to easily include the case of control dependent diffusion. The first general formulation of the SMP for finite horizon controlled stochastic systems in finite dimensions was obtained by Peng in [16]. After this seminal paper, many directions have been followed by many authors. For what concerns ergodic costs, though, the theory is not yet fully developed. As far as we know, the only version of necessary and sufficient condition for optimality goes back to the paper by Kushner [12] in 1978, in which no backward stochastic equation appeared. In that framework the author adopted a martingale solution approach and considered only Markov feedback controls. The system is also assumed to be stable for each control. Under

1991 Mathematics Subject Classification. 60H15, 93E20.

Key words and phrases. Stochastic maximum principle, stochastic ergodic control problems, dissipative systems, backward stochastic differential equation. 
these assumptions, for each stationary Markov control there exists a unique invariant measure $\mu_{u}(\cdot)$ such that the initial cost functional can be rewritten in the following way

$$
\lim _{T \rightarrow \infty} \mathbb{E}_{x_{0}}^{u} \frac{1}{T} \int_{0}^{T} f\left(X_{t}, u\left(X_{t}\right)\right) d t=\int f(x, u(x)) \mu_{u}(d x) .
$$

Using this formulation, Kushner derived a necessary and sufficient condition for $u(\cdot)$ to be optimal, which he called a "dynamic programming like" condition. Let us also mention a recent preprint [5] in which the authors give some sufficient condition for optimality, studying the adjoint Backward SDE, as well as Feller property and exponential ergodicity of the controlled process. As in the present paper the adjoint BSDE is multidimensional in an infinite horizon. The point is that the approach chosen in [5] to prove well posedness of such an equation relies on Girsanov argument and seems to work under commutativity requirements that are satisfied when $n=1$ or when $\sigma$ is constant. Also see [10] for infinite horizon multidimensional BSDEs in the context of linear quadratic stationary optimal control.

Our formulation is fairly general. We do not impose the existence of a limit in the formulation of the cost functional and we consider general progressive controls. Moreover, notice that the convexity assumption on the control actions is a natural choice for the ergodic control problems. Indeed, due to the dissipativity of the system, a spike variation argument is not sufficient to extract useful information on the behaviour of the system at infinity. In the present paper we deduce a version of the maximum principle written in terms of the unique bounded solution to a multidimensional backward SDE on infinite horizon

$$
-d p_{t}=\left[D_{x} b\left(X_{t}, u_{t}\right)^{*} p_{t}+D_{x} \sigma\left(X_{t}, u_{t}\right)^{*} q_{t}-D_{x} f\left(X_{t}, u_{t}\right)\right] d t-q_{t} d W_{t} .
$$

As far as we know, a well-posedness result for backward equations of this form is new. The major difficulty to overcome is the lack of integrability in time of the forcing term of the equation. Due to the hypothesis on the state equation we can guarantee that

$$
\sup _{t \geq 0}\left(\mathbb{E}\left|D_{x} f\left(X_{t}, u_{t}\right)\right|^{r}\right)^{1 / r}<\infty ; \quad \text { for some } r>1 .
$$

Similar equations are studied in the formulation of the SMP for discounted cost functionals in infinite horizon, see e.g. [14], 15]. In that case, though, the spaces in which one is looking for a solution are weighted $L^{2}$-spaces, allowing the solution to explode at infinity in a controlled way. Here, due to the stability of the system, we expect the solution to be bounded up to infinity.

The strategy we employ is mainly built on duality techniques. Via the analysis of a suitable class of perturbed linearized forward eqautions, see equation (37) below, we are able, exploiting their dissipativity, to construct an adjoint process for all positive times. We introduce then a well-suited family of truncated equations and we show the consistency of the family with respect to the varying finite horizon $T>0$, as $T \rightarrow \infty$.

We also propose a second version of maximum principle involving a family of backward equations on finite time horizon $T$ with terminal condition $p_{T}^{T}=0$ that could be verifiable in certain cases, see Remark 4 below.

Once we have a necessary condition for optimality, it is natural to ask also for a sufficient counterpart of it. As in the classical setting, an extra convexity assumption on the Hamiltonian of the system guarantees the required sufficiency.

The paper is structured as follows. In Section 2 we fix the notation and we discuss the main assumptions on the state equation and on the control actions. In Section 3 we study the convex perturbation of the optimal control and we expand the optimal trajectory and cost functional with respect to the perturbation. Section 4 is the core of the paper. Here we introduce the adjoint equation and we present a well-posedness result for it. The main results concerning the necessary and sufficient versions of the SMP are contained in Section 5 and 6.

\section{Preliminaries And assumptions}

Let $(\Omega, \mathcal{F}, \mathbb{P})$ be a complete probability space and $\left(W_{t}\right)_{t \geq 0}$ a standard $d$-dimensional Brownian motion. Throughout the paper we use the natural filtration $\left(\mathcal{F}_{t}\right)_{t \geq 0}$ associated to $W$, augmented in the usual way with the family of $\mathbb{P}$-null sets of $\mathcal{F}$. By $|\cdot|$ we denote the Euclidean norm on $\mathbb{R}^{n}$ and $\|\cdot\|_{2}$ denotes the Hilbert-Schmidt norm on $\mathbb{R}^{n \times n}$.

For any $p \geq 1$ and $T>0$ we define 
- $L^{p}\left(\Omega \times[0, T] ; \mathbb{R}^{n}\right)$, the set of all $\left(\mathcal{F}_{t}\right)$-progressive processes with values in $\mathbb{R}^{n}$ such that

$$
\|X\|_{L^{p}\left(\Omega \times[0, T] ; \mathbb{R}^{n}\right)}=\left(\mathbb{E} \int_{0}^{T}\left|X_{t}\right|^{p} d t\right)^{1 / p}<\infty ;
$$

- $L^{p}\left(\mathbb{R}_{+} ; L^{q}\left(\Omega ; \mathbb{R}^{n}\right)\right)$ the set of all $\left(\mathcal{F}_{t}\right)$-progressive processes with values in $\mathbb{R}^{n}$ with $1 \leq q<$ $+\infty$ such that

and

$$
\|X\|_{L^{p}\left(\mathbb{R}_{+} ; L^{q}\left(\Omega ; \mathbb{R}^{n}\right)\right)}^{p}=\int_{0}^{\infty}\left(\mathbb{E}\left|X_{t}\right|^{q}\right)^{\frac{p}{q}} d t<\infty, \quad \text { for } 1 \leq p<+\infty,
$$

$$
\|X\|_{L^{\infty}\left(\mathbb{R}_{+} ; L^{q}\left(\Omega ; \mathbb{R}^{n}\right)\right)}=\sup _{t \geq 0}\left(\mathbb{E}\left|X_{t}\right|^{q}\right)^{\frac{1}{q}}<\infty .
$$

The aim of this work is to give some necessary (and sufficient) condition for optimality of a controlled system of the form

$$
\left\{\begin{array}{l}
d X_{t}=b\left(X_{t}, u_{t}\right) d t+\sigma\left(X_{t}, u_{t}\right) d W_{t}, \quad t \geq 0 \\
X_{0}=x
\end{array}\right.
$$

when a cost functional of ergodic type has to be minimized. The form of the cost functional slightly differs when considering a liminf or a limsup formulation. We define a truncated cost functional in the following form

$$
J_{T}(u(\cdot))=\mathbb{E} \int_{0}^{T} f\left(X_{t}, u_{t}\right) d t .
$$

Let us denote the two forms in the following way

$$
\begin{gathered}
J^{\inf }(u(\cdot))=\liminf _{T \rightarrow \infty} \frac{1}{T} J_{T}(u(\cdot))=\liminf _{T \rightarrow \infty} \frac{1}{T} \mathbb{E} \int_{0}^{T} f\left(X_{t}, u_{t}\right) d t, \\
J^{\sup }(u(\cdot))=\limsup _{T \rightarrow \infty} \frac{1}{T} J_{T}(u(\cdot))=\limsup _{T \rightarrow \infty} \frac{1}{T} \mathbb{E} \int_{0}^{T} f\left(X_{t}, u_{t}\right) d t .
\end{gathered}
$$

An control process $\bar{u}(\cdot)$ is said to be optimal either if

$$
J^{\inf }(\bar{u}(\cdot))=\inf _{u(\cdot) \in \mathcal{U}} J(u(\cdot)) \quad \text { or } \quad J^{\sup }(\bar{u}(\cdot))=\inf _{u(\cdot) \in \mathcal{U}} J(u(\cdot))
$$

where $\mathcal{U}$ indicates a class of admissible controls. Now we give some assumptions on the state equation and on the control actions.

Hypothesis 1. Assumptions involve three constants $m \geq 0$ and $p>(4 m+2) \vee 4$ and $k>(p-1) / 2$ that we fix now and for the rest of the paper.

(H1) (Controls) $U$ is a closed convex subset of $\mathbb{R}^{l}$. Moreover $u$ is a progressively measurable $U$-valued process. We say that $u$ is an admissible control if it satisfies:

$$
\sup _{t \geq 0} \mathbb{E}\left|u_{t}\right|^{p}<+\infty
$$

(H2) (Polynomial growth) The vector field $b: \mathbb{R}^{n} \times U \rightarrow \mathbb{R}^{n}$ is $\mathcal{B}\left(\mathbb{R}^{n}\right) \otimes \mathcal{B}(U)$-measurable and $\mathcal{C}^{2}$ with respect to $x$ and $u$. There exists $C_{1}>0$ such that

$$
\left|D_{u} b(x, u)\right| \leq C_{1}, \quad x \in \mathbb{R}^{n}, u \in U .
$$

Moreover:

$$
\sup _{u \in U} \sup _{x \in \mathbb{R}^{n}} \frac{\left|D_{x}^{\beta} b(x, u)\right|}{1+|x|^{2 m+1-\beta}+|u|^{1-\beta}}<+\infty, \quad \beta=0,1 .
$$

(H3) (Polynomial growth) The mapping $\sigma: \mathbb{R}^{n} \times U \rightarrow \mathbb{R}^{n \times d}$ is measurable with respect to $\mathcal{B}\left(\mathbb{R}^{n}\right) \otimes \mathcal{B}(U)$. There exists $C_{2}>0$ such that

$$
\left\|D_{u} \sigma(x, u)\right\|_{2} \leq C_{2}, \quad x \in \mathbb{R}^{n}, u \in U .
$$

Moreover it is $\mathcal{C}^{2}$ with respect to $x, u$ and:

$$
\sup _{u \in U} \sup _{x \in \mathbb{R}^{n}} \frac{\left\|D_{x}^{\beta} \sigma(x, u)\right\|_{2}}{1+|x|^{m-\beta}+|u|^{1-\beta}}<+\infty, \quad \beta=0,1 .
$$


(H4)

(Joint dissipativity) There is $c_{p}<0$ such that

$$
\left\langle D_{x} b(x, u) y, y\right\rangle+k\left\|D_{x} \sigma(x, u) y\right\|_{2}^{2} \leq c_{p}|y|^{2}, \quad x, y \in \mathbb{R}^{n}, u \in U
$$

(H5) (Cost) The function $f: \mathbb{R}^{n} \times U \rightarrow \mathbb{R}$ is $\mathcal{B}\left(\mathbb{R}^{n}\right) \otimes \mathcal{B}(U)$-measurable, bounded from below by a constant $f_{0}$, it is differentiable in $x$ and $u$ and

$$
\left|D_{x} f(x, u)\right|+\left|D_{u} f(x, u)\right| \leq C(1+|x|+|u|),
$$

for some $C>0$.

Remark 1. We refer to [4] and [15] for a discussion on the joint monotonicity and on the relation between the growth of $b$ and $\sigma$. Concerning (H5), here we limit ourselves to linear growth for simplicity. A general polynomial growth can be easily achieved.

Remark 2. The choice of $p>4 m+2$ in (H1)-(H4) comes from the interplay between the dissipative behaviour of the system and polynomial growth of the coefficients. Actually this bound can be easily derived from the maximal moment of the state process that we need to estimate in the proofs (see Proposition 1). The condition for $k$ is then the natural one.

We can state the following

Theorem 1. Assume that Hypothesis 1 holds true. Then, for every $x \in \mathbb{R}^{n}$ and every admissible control $u(\cdot)$, equation (5) admits a unique progressively measurable solution for each admissible control. Moreover, the following estimate holds

$$
\mathbb{E}\left|X_{t}\right|^{p} \leq e^{-p \beta t}|x|^{p}+K \sup _{t \geq 0} \mathbb{E}\left|u_{t}\right|^{p}
$$

for some positive constants $K=K\left(p, c_{p}\right)$ and $\beta$.

Proof. Define $\tilde{X}_{t}:=e^{\beta t} X_{t}$ for a positive $\beta$. Then $\tilde{X}$ solves

$$
\left\{\begin{array}{l}
d \tilde{X}_{t}=\beta \tilde{X}_{t}+e^{\beta t} b\left(e^{-\beta t} \tilde{X}_{t}, u_{t}\right) d t+e^{\beta t} \sigma\left(e^{-\beta t} \tilde{X}_{t}, u_{t}\right) d W_{t}, \quad \forall t \geq 0 \\
\tilde{X}_{0}=x
\end{array}\right.
$$

If we call $\tilde{b}_{t}(x, u)=e^{\beta t} b\left(e^{-\beta t} x, u\right)$ and $\tilde{\sigma}_{t}(x, u)=e^{\beta t} \sigma\left(e^{-\beta t} x, u\right)$ then also $\tilde{b}_{t}, \tilde{\sigma}_{t}$ satisfy Hypothesis 1. In particular the joint dissipativity holds with the same constant

$$
\left\langle\tilde{b}_{t}(x, u)-\tilde{b}_{t}(y, u), x-y\right\rangle+\frac{p-1}{2}\left\|\tilde{\sigma}_{t}(x, u)-\tilde{\sigma}_{t}(y, u)\right\|_{2}^{2} \leq c_{p}|x-y|^{2} .
$$

Let $p \geq 2$, denote $p=2 q$ and $\tilde{a}=\tilde{\sigma}(x, u)^{*} \tilde{\sigma}(x, u)$ (we omit the time dependence $\tilde{\sigma}=\tilde{\sigma}_{t}$ when it is clear). We apply the Itô formula to the function $f(x)=|x|^{2 q}$ to get

$$
\begin{aligned}
& \mathbb{E}\left|\tilde{X}_{t}\right|^{2 q}=|x|^{2 q}+2 q \mathbb{E} \int_{0}^{t}\left|\tilde{X}_{s}\right|^{2(q-1)}\left(\left\langle\tilde{X}_{s}, \tilde{b}\left(\tilde{X}_{s}, u_{s}\right)\right\rangle+\frac{1}{2}\left\|\tilde{\sigma}\left(\tilde{X}_{s}, u_{s}\right)\right\|_{2}^{2}\right) d s \\
& \quad+2 q \beta \mathbb{E} \int_{0}^{t}\left|\tilde{X}_{s}\right|^{2 q} d s+2 q(q-1) \mathbb{E} \int_{0}^{t}\left|\tilde{X}_{s}\right|^{2(q-2)} \operatorname{Tr}\left\{\tilde{a}_{s}\left(\tilde{X}_{s} \otimes \tilde{X}_{s}\right)\right\} d s \\
& \leq|x|^{2 q}+2 q \mathbb{E} \int_{0}^{t}\left|\tilde{X}_{s}\right|^{2(q-1)}\left(\left\langle\tilde{X}_{s}, \tilde{b}\left(\tilde{X}_{s}, u_{s}\right)\right\rangle+\left(q-\frac{1}{2}\right)\left\|\tilde{\sigma}\left(\tilde{X}_{s}, u_{s}\right)\right\|_{2}^{2}\right) d s+2 q \beta \mathbb{E} \int_{0}^{t}\left|\tilde{X}_{s}\right|^{2 q} d s \\
& \leq|x|^{2 q}+2 q \mathbb{E} \int_{0}^{t}\left|\tilde{X}_{s}\right|^{2(q-1)}\left(\left\langle\tilde{X}_{s}, \tilde{b}\left(\tilde{X}_{s}, u_{s}\right)-\tilde{b}\left(0, u_{s}\right)\right\rangle+\left(q-\frac{1}{2}\right)(1+\varepsilon)\left\|\tilde{\sigma}\left(\tilde{X}_{s}, u_{s}\right)-\tilde{\sigma}\left(0, u_{s}\right)\right\|_{2}^{2}\right) d s
\end{aligned}
$$




$$
\begin{aligned}
& +2 q \mathbb{E} \int_{0}^{t}\left|\tilde{X}_{s}\right|^{2(q-1)}\left(\left\langle\tilde{X}_{s}, \tilde{b}\left(0, u_{s}\right)\right\rangle+c_{\varepsilon}\left\|\tilde{\sigma}\left(0, u_{s}\right)\right\|_{2}^{2}\right) d s+2 q \beta \mathbb{E} \int_{0}^{t}\left|\tilde{X}_{s}\right|^{2 q} d s \\
\leq & |x|^{2 q}+2 q\left(c_{r}+\beta+\frac{\delta}{2}\right) \mathbb{E} \int_{0}^{t}\left|\tilde{X}_{s}\right|^{2 q} d s \quad \quad(\text { with } r=2 q(1+\varepsilon)-\varepsilon) \\
& +2 q \mathbb{E} \int_{0}^{t}\left|\tilde{X}_{s}\right|^{2(q-1)}\left(\frac{1}{2 \delta}\left|\tilde{b}\left(0, u_{s}\right)\right|^{2}+c_{\varepsilon}\left\|\tilde{\sigma}\left(0, u_{s}\right)\right\|_{2}^{2}\right) d s \\
\leq & |x|^{2 q}+2 q\left(c_{r}+\beta+\frac{\delta}{2}+c_{\delta} \delta^{q /(q-1)}\right) \mathbb{E} \int_{0}^{t}\left|\tilde{X}_{s}\right|^{2 q} d s \\
& +2 q \mathbb{E} \int_{0}^{t} e^{-2 q \beta t}\left(\frac{1}{2^{q} \delta^{q+1} q}+\frac{c_{\varepsilon}}{\delta^{q}}\right)\left|u_{s}\right|^{2 q} d s,
\end{aligned}
$$

where we employed joint dissipativity for the process $\tilde{X}$, we repeatedly used weighted Young inequality and in the end the growth condition on the coefficients. Choosing $\beta$ and $\delta$ small enough, thanks to Hypothesis (H1) we end up with the following estimate

$$
\begin{aligned}
\mathbb{E}\left|X_{t}\right|^{2 q} & \leq e^{-2 q \beta t}|x|^{2 q}+C \int_{0}^{t} e^{-2 q \beta(t-s)} \mathbb{E}\left|u_{s}\right|^{2 q} d s \\
& \leq e^{-2 q \beta t}|x|^{2 q}+C \sup _{t \geq 0} \mathbb{E}\left|u_{t}\right|^{2 q} .
\end{aligned}
$$

Notice that, taking the supremum on both sides we also have that

$$
\sup _{t \geq 0} \mathbb{E}\left|X_{t}\right|^{2 q} \leq C\left(|x|^{2 q}+1\right)
$$

and the claim is proved.

\section{Perturbation of the controls}

When considering ergodic control problems we can not expect to gain information by the use of local in time perturbations of the optimal control.

More precisely, let $u^{i}(\cdot), i=1,2$ are admissible controls with $u_{t}^{1}=u_{t}^{2}$ for all $t>T_{0}$. If one denotes by $X^{i}, i=1,2$ the corresponding states then by the dissipativity assumption (H4) one gets $\mathbb{E}\left|X_{t}^{1}-X_{t}^{2}\right|^{2} \rightarrow 0$ for $t>T_{0}$ exponentially fast (let us say with exponential decay $\varepsilon$ ). Consequently (assume for a moment that $f$ is Lipschitz)

$$
\begin{aligned}
\left|J\left(u^{1}(\cdot)\right)-J\left(u^{2}(\cdot)\right)\right| & =\lim _{T \rightarrow \infty} \frac{1}{T} \mathbb{E} \int_{0}^{T}\left|f\left(X_{t}^{1}, u_{t}^{1}\right)-f\left(X_{t}^{2}, u_{t}^{2}\right)\right| d t \\
& =\lim _{T \rightarrow \infty} \frac{1}{T} \mathbb{E} \int_{T_{0}}^{T}\left|f\left(X_{t}^{1}, u_{t}^{1}\right)-f\left(X_{t}^{2}, u_{t}^{1}\right)\right| d t \\
& \leq C \lim _{T \rightarrow \infty} \frac{1}{T} \int_{T_{0}}^{T} e^{-\varepsilon t} d t=0,
\end{aligned}
$$

This is the reason for considering the perturbations which act on the system up to infinity. Notice that it is crucial to require that $U$ is convex.

Let then $\bar{u}(\cdot)$ be an optimal control for the ergodic control problem (91) and denote the corresponding state process as $\bar{X}$. For $\theta \in(0,1]$ and $u(\cdot)$ admissible control define $u^{\theta}$ as a convex combination by $u^{\theta}(\cdot):=(1-\theta) \bar{u}(\cdot)+\theta u(\cdot)=\bar{u}(\cdot)+\theta v(\cdot)$, where $v(\cdot):=u(\cdot)-\bar{u}(\cdot)$. Then $u^{\theta}(\cdot)$ is admissible and the corresponding state is denoted by $X^{\theta}$.

Lemma 1. Under Hypothesis 1 the following holds

$$
\sup _{t \geq 0} \mathbb{E}\left|X_{t}^{\theta}-\bar{X}_{t}\right|^{p} \leq C \theta^{2} \sup _{t \geq 0} \mathbb{E}\left|v_{t}\right|^{p} .
$$

Where $C$ only depends on the constants appearing in Hypothesis 1 .

Proof. Denote $\Delta X_{t}^{\theta}:=X_{t}^{\theta}-\bar{X}_{t}$ and write the corresponding equation

$$
\Delta X_{t}^{\theta}=\int_{0}^{t}\left[b\left(X_{s}^{\theta}, u_{s}^{\theta}\right)-b\left(\bar{X}_{s}, \bar{u}_{s}\right)\right] d s+\int_{0}^{t}\left[\sigma\left(X_{s}^{\theta}, u_{s}^{\theta}\right)-\sigma\left(\bar{X}_{s}, \bar{u}_{s}\right)\right] d W_{s} .
$$


Following the technique developed in the proof of Theorem 1 we define $\Delta \tilde{X}_{t}^{\theta}:=e^{\beta t} \Delta X_{t}^{\theta}$ for a positive $\beta$. Then the Itô formula gives

$$
\begin{aligned}
\mathbb{E} \mid & \left.\Delta \tilde{X}_{t}^{\theta}\right|^{2 q} \\
\leq & 2 q \mathbb{E} \int_{0}^{t}\left|\Delta \tilde{X}_{s}^{\theta}\right|^{2(q-1)}\left[\left\langle\tilde{b}\left(\tilde{X}_{s}^{\theta}, u_{s}^{\theta}\right)-\tilde{b}\left(\tilde{\bar{X}}_{s}, u_{s}^{\theta}\right), \Delta \tilde{X}_{s}^{\theta}\right\rangle+\left(q-\frac{1}{2}\right)(1+\varepsilon)\left\|\tilde{\sigma}\left(\tilde{X}_{s}^{\theta}, u_{s}^{\theta}\right)-\tilde{\sigma}\left(\tilde{\bar{X}}_{s}, u_{s}^{\theta}\right)\right\|_{2}^{2}\right] d s \\
& +2 q \beta \mathbb{E} \int_{0}^{t}\left|\Delta \tilde{X}_{s}^{\theta}\right|^{2 q} d s+2 q \mathbb{E} \int_{0}^{t}\left|\Delta \tilde{X}_{s}^{\theta}\right|^{2(q-1)}\left\langle\int_{0}^{1} D_{u} \tilde{b}\left(\tilde{\bar{X}}_{s}, \bar{u}_{s}+\lambda \theta v_{s}\right) \theta v_{s} d \lambda, \Delta \tilde{X}_{s}^{\theta}\right\rangle d s \\
& +2 q c_{\varepsilon} \mathbb{E} \int_{0}^{t}\left|\Delta \tilde{X}_{s}^{\theta}\right|^{2(q-1)}\left\|\int_{0}^{1} D_{u} \tilde{\sigma}\left(\tilde{\bar{X}}_{s}, \bar{u}_{s}+\lambda \theta v_{s}\right) \theta v_{s} d \lambda\right\|_{2}^{2} d s \\
\leq & \left.2 q\left(c_{r}+\beta+\frac{\delta}{2}\right) \int_{0}^{t}\left|\Delta \tilde{X}_{s}^{\theta}\right|^{2 q} d s+2 q \theta^{2} \mathbb{E} \int_{0}^{t}\left|\Delta \tilde{X}_{s}^{\theta}\right|^{2(q-1)} \frac{e^{2 \beta s}}{2 \delta}\left|v_{s}\right|^{2} d s \quad \quad \text { with } r=2 q(1+\varepsilon)-\varepsilon\right) \\
& +2 q c_{\varepsilon} \theta^{2} \mathbb{E} \int_{0}^{t}\left|\Delta \tilde{X}_{s}^{\theta}\right|^{2(q-1)} e^{2 \beta s}\left|v_{s}\right|^{2} d s \\
\leq & 2 q\left(c_{r}+\beta+\frac{\delta}{2}+c_{\delta} \theta^{2} \delta^{q /(q-1)}\right) \int_{0}^{t}\left|\Delta \tilde{X}_{s}^{\theta}\right|^{2 q} d s \\
& +2 q \theta^{2} \mathbb{E} \int_{0}^{t} e^{2 q \beta s}\left(\frac{1}{2^{q} \delta^{q+1}}+\frac{c_{\varepsilon}}{\delta^{q}}\right)\left|v_{s}\right|^{2 q} d s .
\end{aligned}
$$

Where we used the joint dissipativity and weighted Young inequality, for every $\delta>0$. Choosing $\beta$, $\delta$ small enough, from the boundedness of $\sup _{s \geq 0} \mathbb{E}\left|v_{s}\right|^{2 q}$ we get

$$
\mathbb{E}\left|\Delta X_{t}^{\theta}\right|^{2 q} \leq C \theta^{2} \mathbb{E} \int_{0}^{t} e^{-2 q \beta(t-s)} d s .
$$

The result follows by taking the supremum in time and finally by sending $\theta \rightarrow 0_{+}$.

Now we introduce the first variation equation of the system. Notice that in the equation appears the derivative of the coefficients with respect to the control, which are bounded due to our assumptions.

$$
\left\{\begin{array}{l}
d Y_{t}=\left[D_{x} b\left(\bar{X}_{t}, \bar{u}_{t}\right) Y_{t}+D_{u} b\left(\bar{X}_{t}, \bar{u}_{t}\right) v_{t}\right] d t+\left[D_{x} \sigma\left(\bar{X}_{t}, \bar{u}_{t}\right) Y_{t}+D_{u} \sigma\left(\bar{X}_{t}, \bar{u}_{t}\right) v_{t}\right] d W_{t}, \\
Y_{0}=0,
\end{array}\right.
$$

Lemma 2. Under Hypothesis 1, the first variation equation (21) admits a unique adapted solution. Moreover the following estimate holds true

$$
\mathbb{E}\left|Y_{t}\right|^{p} \leq K \sup _{s \in[0, t]} \mathbb{E}\left|v_{s}\right|^{p} .
$$

where again $K$ only depends on the constants appearing in Hypothesis 1 .

In particular, $\sup _{t \geq 0} \mathbb{E}\left|Y_{t}\right|^{p} \leq K \sup _{t \geq 0} \mathbb{E}\left|v_{t}\right|^{p}<+\infty$.

Proof. The proof goes through by the same technique adopted in Theorem 11. What is crucial here is the uniform boundedness of $D_{u} b(x, u)$ and $D_{u} \sigma(x, u)$, along with the assumption (H1) on admissible controls.

The following lemma is fundamental in order to obtain the right expansion of the cost functional with respect to the control.

Proposition 1. Under our assumptions the process $\hat{X}^{\theta}$ defined as

$$
\hat{X}_{t}^{\theta}=\frac{X_{t}^{\theta}-\bar{X}_{t}}{\theta}-Y_{t}
$$

satisfies

$$
\lim _{\theta \rightarrow 0_{+}} \sup _{t \geq 0} \mathbb{E}\left|\hat{X}_{t}^{\theta}\right|^{2}=0
$$


Proof. The equation for $\hat{X}^{\theta}$ reads

$$
\begin{aligned}
d \hat{X}_{t}^{\theta}= & \frac{1}{\theta}\left[b\left(X_{t}^{\theta}, u_{t}^{\theta}\right)-b\left(\bar{X}_{t}, \bar{u}_{t}\right)-\theta D_{x} b\left(\bar{X}_{t}, \bar{u}_{t}\right) Y_{t}-\theta D_{u} b\left(\bar{X}_{t}, \bar{u}_{t}\right) v_{t}\right] d t \\
& +\frac{1}{\theta}\left[\sigma\left(X_{t}^{\theta}, u_{t}^{\theta}\right)-\sigma\left(\bar{X}_{t}, \bar{u}_{t}\right)-\theta D_{x} \sigma\left(\bar{X}_{t}, \bar{u}_{t}\right) Y_{t}-\theta D_{u} \sigma\left(\bar{X}_{t}, \bar{u}_{t}\right) v_{t}\right] d W_{t} \\
= & \frac{1}{\theta}\left[b\left(\bar{X}_{t}+\theta\left(Y_{t}+\hat{X}_{t}^{\theta}\right), \bar{u}_{t}+\theta v_{t}\right)-b\left(\bar{X}_{t}, \bar{u}_{t}\right)-\theta D_{x} b\left(\bar{X}_{t}, \bar{u}_{t}\right) Y_{t}-\theta D_{u} b\left(\bar{X}_{t}, \bar{u}_{t}\right) v_{t}\right] d t \\
& +\frac{1}{\theta}\left[\sigma\left(\bar{X}_{t}+\theta\left(Y_{t}+\hat{X}_{t}^{\theta}\right), \bar{u}_{t}+\theta v_{t}\right)-\sigma\left(\bar{X}_{t}, \bar{u}_{t}\right)-\theta D_{x} \sigma\left(\bar{X}_{t}, \bar{u}_{t}\right) Y_{t}-\theta D_{u} \sigma\left(\bar{X}_{t}, \bar{u}_{t}\right) v_{t}\right] d W_{t},
\end{aligned}
$$

with $\hat{X}_{0}^{\theta}=0$ as initial condition. Further, by Taylor expansion we have that

$$
\begin{aligned}
d \hat{X}_{t}^{\theta}= & \int_{0}^{1} D_{x} b\left(\bar{X}_{t}+\lambda \theta\left(Y_{t}+\hat{X}_{t}^{\theta}\right), \bar{u}_{t}+\lambda \theta v_{t}\right) \hat{X}_{t}^{\theta} d \lambda d t \\
& +\int_{0}^{1}\left[D_{x} b\left(\bar{X}_{t}+\lambda \theta\left(Y_{t}+\hat{X}_{t}^{\theta}\right), \bar{u}_{t}+\lambda \theta v_{t}\right)-D_{x} b\left(\bar{X}_{t}, \bar{u}_{t}\right)\right] Y_{t} d \lambda d t \\
& +\int_{0}^{1}\left[D_{u} b\left(\bar{X}_{t}+\lambda \theta\left(Y_{t}+\hat{X}_{t}^{\theta}\right), \bar{u}_{t}+\lambda \theta v_{t}\right)-D_{u} b\left(\bar{X}_{t}, \bar{u}_{t}\right)\right] v_{t} d \lambda d t \\
& +\int_{0}^{1} D_{x} \sigma\left(\bar{X}_{t}+\lambda \theta\left(Y_{t}+\hat{X}_{t}^{\theta}\right), \bar{u}_{t}+\lambda \theta v_{t}\right) \hat{X}_{t}^{\theta} d \lambda d W_{t} \\
& +\int_{0}^{1}\left[D_{x} \sigma\left(\bar{X}_{t}+\lambda \theta\left(Y_{t}+\hat{X}_{t}^{\theta}\right), \bar{u}_{t}+\lambda \theta v_{t}\right)-D_{x} \sigma\left(\bar{X}_{t}, \bar{u}_{t}\right)\right] Y_{t} d \lambda d W_{t} \\
& +\int_{0}^{1}\left[D_{u} \sigma\left(\bar{X}_{t}+\lambda \theta\left(Y_{t}+\hat{X}_{t}^{\theta}\right), \bar{u}_{t}+\lambda \theta v_{t}\right)-D_{u} \sigma\left(\bar{X}_{t}, \bar{u}_{t}\right)\right] v_{t} d \lambda d W_{t}
\end{aligned}
$$

To keep the notation simple, we rewrite the above equation as

$$
d \hat{X}_{t}^{\theta}=\left(A_{t}^{x} \hat{X}_{t}^{\theta}+A_{t}^{y} Y_{t}+A_{t}^{v} v_{t}\right) d t+\left(B_{t}^{x} \hat{X}_{t}^{\theta}+B_{t}^{y} Y_{t}+B_{t}^{v} v_{t}\right) d W_{t}
$$

where we have kept the order of the terms from the previous equation.

Now apply the Itô formula to $e^{\beta t}\left|\hat{X}_{t}^{\theta}\right|^{2}$ to get

$$
\begin{aligned}
\mathbb{E}\left(e^{\beta t}\left|\hat{X}_{t}^{\theta}\right|^{2}\right) & =2 \mathbb{E} \int_{0}^{t} e^{\beta s}\left\langle A_{s}^{x} \hat{X}_{s}^{\theta}+A_{s}^{y} Y_{s}+A_{s}^{v} v_{s}, \hat{X}_{s}^{\theta}\right\rangle d s \\
& +\mathbb{E} \int_{0}^{t} e^{\beta s}\left\|B_{s}^{x} \hat{X}_{s}^{\theta}+B_{s}^{y} Y_{s}+B_{s}^{v} v_{s}\right\|_{2}^{2} d s+\beta \mathbb{E} \int_{0}^{t} e^{\beta s}\left|\hat{X}_{s}^{\theta}\right|^{2} d s .
\end{aligned}
$$

By the joint dissipativity assumption (H4) in Hypothesis 1 we have

$$
2\left\langle A_{s}^{x} \hat{X}_{s}^{\theta}, \hat{X}_{s}^{\theta}\right\rangle+2 k\left\|B_{s}^{x} \hat{X}_{s}^{\theta}\right\|^{2}+\beta\left|\hat{X}_{s}^{\theta}\right|^{2}<0
$$

for some $k>1 / 2$ and $\beta$ small enough.

Thus, repeating the same computations as in the proof of Theorem 1, we get the following intermediate estimate

$$
\mathbb{E}\left|\hat{X}_{t}^{\theta}\right|^{2} \leq C \int_{0}^{t} e^{-\beta(t-s)} \mathbb{E}\left(\left|A_{s}^{y} Y_{s}\right|^{2}+\left|A_{s}^{v} v_{s}\right|^{2}+\left|B_{s}^{y} Y_{s}\right|^{2}+\left|B_{s}^{v} v_{s}\right|^{2}\right) d s .
$$

Now we show how to treat the first term in (25). The estimate of the remaining ones goes along similar lines.

We fix $\alpha$ with $p /(p-2)<\alpha<p /(4 m)$, if $m \geq 1$, or $\alpha=2$, if $m=0$. Recall that $p>4 m+2$ and notice that, this way, denoting by $\alpha^{\prime}$ the conjugate of $\alpha$ (that is $1 / \alpha+1 / \alpha^{\prime}=1$ ) then $2 \alpha^{\prime}<p$ $4 m \alpha<p$ and $2 \alpha<p$. First we start by observing that by Hölder inequality and by (22) we have 
that for any $\alpha>1$.

$$
\begin{aligned}
& \int_{0}^{t} e^{-\beta(t-s)} \mathbb{E}\left|A_{s}^{y} Y_{s}\right|^{2} d s \\
& =\int_{0}^{t} e^{-\beta(t-s)} \mathbb{E}\left|\int_{0}^{1}\left[D_{x} b\left(\bar{X}_{s}+\lambda \theta\left(Y_{s}+\hat{X}_{s}^{\theta}\right), \bar{u}_{s}+\lambda \theta v_{s}\right)-D_{x} b\left(\bar{X}_{s}, \bar{u}_{s}\right)\right] Y_{s} d \lambda\right|^{2} d s \\
& \leq \int_{0}^{t} e^{-\beta(t-s)}\left(\int_{0}^{1} \mathbb{E}\left|D_{x} b\left(\bar{X}_{s}+\lambda \theta\left(Y_{s}+\hat{X}_{s}^{\theta}\right), \bar{u}_{s}+\lambda \theta v_{s}\right)-D_{x} b\left(\bar{X}_{s}, \bar{u}_{s}\right)\right|^{2 \alpha} d \lambda\right)^{\frac{1}{\alpha}} \cdot\left(\mathbb{E}\left|Y_{s}\right|^{2 \alpha^{\prime}}\right)^{\frac{1}{\alpha^{\prime}}} d s
\end{aligned}
$$

Since $2 \alpha^{\prime}<p$, using Lemma 2 to estimate $\sup _{s \in \mathbb{R}^{+}} \mathbb{E}\left|Y_{s}\right|^{2 \alpha^{\prime}}$ :

$$
\begin{aligned}
& \int_{0}^{t} e^{-\beta(t-s)} \mathbb{E}\left|A_{s}^{y} Y_{s}\right|^{2} d s \\
& \leq C \int_{0}^{t} e^{-\beta(t-s)}\left(\int_{0}^{1} \mathbb{E}\left|D_{x} b\left(\bar{X}_{s}+\lambda \theta\left(Y_{s}+\hat{X}_{s}^{\theta}\right), \bar{u}_{s}+\lambda \theta v_{s}\right)-D_{x} b\left(\bar{X}_{s}, \bar{u}_{s}\right)\right|^{2 \alpha} d \lambda\right)^{\frac{1}{\alpha}} d s . \\
& \leq C \int_{0}^{t} e^{-\beta(t-s)}\left(\int_{0}^{1} \mathbb{E}\left|D_{x} b\left(\bar{X}_{s}+\lambda \theta\left(Y_{s}+\hat{X}_{s}^{\theta}\right), \bar{u}_{s}+\lambda \theta v_{s}\right)-D_{x} b\left(\bar{X}_{s}, \bar{u}_{s}+\lambda \theta v_{s}\right)\right|^{2 \alpha} d \lambda\right)^{\frac{1}{\alpha}} d s \\
& \quad+C \int_{0}^{t} e^{-\beta(t-s)}\left(\int_{0}^{1} \mathbb{E}\left|D_{x} b\left(\bar{X}_{s}, \bar{u}_{s}+\lambda \theta v_{s}\right)-D_{x} b\left(\bar{X}_{s}, \bar{u}_{s}\right)\right|^{2 \alpha} d \lambda\right)^{\frac{1}{\alpha}} d s .
\end{aligned}
$$

We prove convergence of the first term, being the second similar (and easier).

Due to Hypothesis 1, the gradients $D_{x} b$ are locally Lipschitz functions with respect to $x$, so that for all $R>0$ there exists $C_{R}$ such that $D_{x} b$ is Lipschitz with constant $C_{R}$ in the ball of radius $R$. For each $t$ and $\theta$ we define the sets

$$
A_{t, \theta}(R)=\left\{w \in \Omega:\left|\bar{X}_{t}\right|>R\right\} \cup\left\{w \in \Omega:\left|X_{t}^{\theta}\right|>R\right\} .
$$

By Chebyshev inequality we know that

$$
\mathbb{P}\left(A_{t, \theta}(R)\right) \leq \frac{\mathbb{E}\left|\bar{X}_{t}\right|^{2}}{R^{2}}+\frac{\mathbb{E}\left|X_{t}^{\theta}\right|^{2}}{R^{2}} \leq \frac{C}{R^{2}}, \quad \forall t, \forall \theta .
$$

Denoting for simplicity $\left.X_{s}^{\lambda}=\bar{X}_{s}+\lambda \theta\left(Y_{s}+\hat{X}_{s}\right)\right)=(1-\lambda) \bar{X}_{s}+\lambda X_{s}^{\theta}$ we have

$$
\begin{aligned}
& \int_{0}^{t} e^{-\beta(t-s)}\left(\int_{0}^{1} \mathbb{E}\left|D_{x} b\left(X_{s}^{\lambda}, \bar{u}_{s}+\lambda \theta v_{s}\right)-D_{x} b\left(\bar{X}_{s}, \bar{u}_{s}+\lambda \theta v_{s}\right)\right|^{2 \alpha} d \lambda\right)^{\frac{1}{\alpha}} d s \\
& \leq C \int_{0}^{t} e^{-\beta(t-s)}\left(\int_{0}^{1} \int_{A_{s, \theta}(R)}\left|D_{x} b\left(X_{s}^{\lambda}, \bar{u}_{s}+\lambda \theta v_{s}\right)-D_{x} b\left(\bar{X}_{s}, \bar{u}_{s}+\lambda \theta v_{s}\right)\right|^{2 \alpha} d \mathbb{P} d \lambda\right)^{\frac{1}{\alpha}} d s \\
&+C \int_{0}^{t} e^{-\beta(t-s)}\left(\int_{0}^{1} \int_{A_{s, \theta}^{c}(R)}\left|D_{x} b\left(X_{s}^{\lambda}, \bar{u}_{s}+\lambda \theta v_{s}\right)-D_{x} b\left(\bar{X}_{s}, \bar{u}_{s}+\lambda \theta v_{s}\right)\right|^{2 \alpha} d \mathbb{P} d \lambda\right)^{\frac{1}{\alpha}} d s \\
& \leq C \int_{0}^{t} e^{-\beta(t-s)}\left(\int_{0}^{1} \mathbb{P}\left(A_{s, \theta}(R)\right)^{\frac{\delta}{1+\delta}} \mathbb{E}\left|D_{x} b\left(X_{s}^{\lambda}, \bar{u}_{s}+\lambda \theta v_{s}\right)-D_{x} b\left(\bar{X}_{s}, \bar{u}_{s}+\lambda \theta v_{s}\right)\right|^{2 \alpha(1+\delta)} d \lambda\right)^{\frac{1}{\alpha(1+\delta)}} d s \\
&+C \int_{0}^{t} e^{-\beta(t-s)} C_{R}^{\frac{1}{\alpha}}\left(\mathbb{E}\left|X_{s}^{\theta}-\bar{X}_{s}\right|^{2 \alpha}\right)^{\frac{1}{\alpha}} d s,
\end{aligned}
$$

where $\delta>0$ is such that $4 m \alpha(1+\delta) \leq p$.

Fixed $\varepsilon>0$ we know by (29) that there exists $R$ large enough so that $\mathbb{P}\left(A_{s, \theta}(R)\right) \leq \varepsilon$. Moreover by Hypothesis 1, Theorem 1 and Lemma 1.

$$
\mathbb{E}\left|D_{x} b\left(X_{s}^{\lambda}, \bar{u}_{s}+\lambda \theta v_{s}\right)-D_{x} b\left(\bar{X}_{s}, \bar{u}_{s}+\lambda \theta v_{s}\right)\right|^{2 \alpha(1+\delta)} \leq C\left(\mathbb{E}\left|X_{s}^{\lambda}\right|^{4 m \alpha(1+\delta)}+\mathbb{E}\left|\bar{X}_{s}\right|^{4 m \alpha(1+\delta)}\right) \leq C,
$$


(if $m=0$ the above relation is straight forward). Thus the first of the two integrals in the last two lines in (30) can be estimated, for $R$ large enough and all $\theta, \lambda$ in [0,1], by $C \varepsilon^{\delta /\left[\alpha(1+\delta)^{2}\right]}$.

Moreover, due to Lemma 1 we have that $\sup _{t>0} \mathbb{E}\left|X_{t}^{\theta}-\bar{X}_{t}\right|^{p} \rightarrow 0$ as $\theta \rightarrow 0$.

Combining the two estimates above we have:

$$
\sup _{t \geq 0} \int_{0}^{t} e^{-\beta(t-s)} \mathbb{E}\left|A_{s}^{y} Y_{s}\right|^{2} d s \rightarrow 0 \text { as } \theta \rightarrow 0 .
$$

Repeating the argument for all the terms in (25) we get the required result.

Remark 3. Notice that we estimate only the second moment of the error term, uniformly in time. Nevertheless, estimate of higer moments of the the state and first variation process are needed in order to complete the proof. More precisely, we can tune the value of $\alpha$ in (26) in order to minimize the maximal moment of the state equation we need to control. Indeed, the growth of the first term is

$$
\mathbb{E}\left|D_{x} b\left(\bar{X}_{s}+\lambda \theta\left(Y_{s}+\hat{X}_{s}^{\theta}\right), \bar{u}_{s}+\lambda \theta v_{s}\right)-D_{x} b\left(\bar{X}_{s}, \bar{u}_{s}\right)\right|^{2 \alpha} \leq C \mathbb{E}\left|\bar{X}_{s}\right|^{4 m \alpha} .
$$

So that, $4 m \alpha=2 \alpha^{\prime}=2 \frac{\alpha}{\alpha-1}$, from which $\alpha=\frac{2 m+1}{2 m}$. The maximal moment is then $p=4 m \alpha=$ $2(2 m+1)$, which is the one appearing in Hypothesis 1 .

3.1. Perturbation of the cost. Due to the hypotheses on the admissible controls and the estimate (14) the cost is well posed:

$$
\liminf _{T \rightarrow \infty} \frac{1}{T} \mathbb{E} \int_{0}^{T} f\left(X_{t}, u_{t}\right) d t \leq K\left[1+\sup _{t \geq 0} \mathbb{E}\left|X_{t}\right|^{2}+\sup _{t \geq 0} \mathbb{E}\left|u_{t}\right|^{2}\right]<\infty .
$$

The same is true for the limsup formulation. The expansion of the functional with respect to a convex perturbation of the control is given in the following

Lemma 3. Let $\bar{u}$ be an optimal control and let $u$ be any admissible control. Letting $v=u-\bar{u}$ and using the above notation the following holds:

$$
\lim _{\theta \rightarrow 0_{+}} \frac{J^{\inf }(\bar{u}(\cdot)+\theta v(\cdot))-J^{\inf }(\bar{u}(\cdot))}{\theta} \leq \limsup _{T \rightarrow \infty} \frac{1}{T} \mathbb{E} \int_{0}^{T}\left[\left\langle D_{x} f\left(\bar{X}_{t}, \bar{u}_{t}\right), Y_{t}\right\rangle_{\mathbb{R}^{n}}+\left\langle D_{u} f\left(\bar{X}_{t}, \bar{u}_{t}\right), v_{t}\right\rangle_{\mathbb{R}^{l}}\right] d t,
$$

and

$\lim _{\theta \rightarrow 0_{+}} \frac{J^{\sup }(\bar{u}(\cdot)+\theta v(\cdot))-J^{\sup }(\bar{u}(\cdot))}{\theta} \leq \limsup _{T \rightarrow \infty} \frac{1}{T} \mathbb{E} \int_{0}^{T}\left[\left\langle D_{x} f\left(\bar{X}_{t}, \bar{u}_{t}\right), Y_{t}\right\rangle_{\mathbb{R}^{n}}+\left\langle D_{u} f\left(\bar{X}_{t}, \bar{u}_{t}\right), v_{t}\right\rangle_{\mathbb{R}^{l}}\right] d t$.

Proof. We prove the first relation. The proof of the second one goes along the same lines. Let us compute

$$
\begin{aligned}
& \frac{J_{T}(\bar{u}(\cdot)+\theta v(\cdot))-J_{T}(\bar{u}(\cdot))}{\theta}=\frac{1}{\theta} \mathbb{E} \int_{0}^{T}\left[f\left(X_{t}^{\theta}, \bar{u}_{t}+\theta v_{t}\right)-f\left(\bar{X}_{t}, \bar{u}_{t}\right)\right] d t \\
& =\mathbb{E} \int_{0}^{T} \int_{0}^{1} D_{x} f\left(\bar{X}_{t}+\lambda\left(X_{t}^{\theta}-\bar{X}_{t}\right), \bar{u}_{t}+\lambda \theta v_{t}\right)\left(\hat{X}_{t}^{\theta}+Y_{t}\right) d \lambda d t \\
& \quad+\mathbb{E} \int_{0}^{T} \int_{0}^{1} D_{u} f\left(\bar{X}_{t}+\lambda\left(X_{t}^{\theta}-\bar{X}_{t}\right), \bar{u}_{t}+\lambda \theta v_{t}\right) v_{t} d \lambda d t .
\end{aligned}
$$

Passing to the ergodic liminf cost functional (2) we have that

$$
\begin{aligned}
& \frac{J^{\inf }(\bar{u}(\cdot)+\theta v(\cdot))-J^{\inf }(\bar{u}(\cdot))}{\theta}=\frac{1}{\theta}\left[\liminf _{T \rightarrow \infty} \frac{1}{T} J_{T}(\bar{u}(\cdot)+\theta v(\cdot))-\liminf _{T \rightarrow \infty} \frac{1}{T} J_{T}(\bar{u}(\cdot))\right] \\
& \leq \limsup _{T \rightarrow \infty} \frac{1}{T}\left[\frac{J_{T}(\bar{u}(\cdot)+\theta v(\cdot))-J_{T}(\bar{u}(\cdot))}{\theta}\right] \\
& =\limsup _{T \rightarrow \infty} \frac{1}{T} \mathbb{E} \int_{0}^{T} \int_{0}^{1}\left\langle D_{x} f\left(\bar{X}_{t}+\lambda\left(X_{t}^{\theta}-\bar{X}_{t}\right), \bar{u}_{t}+\lambda \theta v_{t}\right), \hat{X}_{t}^{\theta}+Y_{t}\right\rangle d \lambda d t \\
& \quad+\limsup _{T \rightarrow \infty} \frac{1}{T} \mathbb{E} \int_{0}^{T} \int_{0}^{1}\left\langle D_{u} f\left(\bar{X}_{t}+\lambda\left(X_{t}^{\theta}-\bar{X}_{t}\right), \bar{u}_{t}+\lambda \theta v_{t}\right), v_{t}\right\rangle_{U} d \lambda d t,
\end{aligned}
$$


where we used that $\lim \sup \left(a_{n}\right)-\lim \sup \left(b_{n}\right) \leq \limsup \left(a_{n}-b_{n}\right)$, for $\left(a_{n}\right)_{n \geq 1}$ and $\left(b_{n}\right)_{n \geq 1}$ two general real sequences. The extra term can be estimated by

$$
\begin{aligned}
\limsup _{T \rightarrow \infty} & \frac{1}{T} \mathbb{E} \int_{0}^{T} \int_{0}^{1}\left\langle D_{x} f\left(\bar{X}_{t}+\lambda\left(X_{t}^{\theta}-\bar{X}_{t}\right), \bar{u}_{t}+\lambda \theta v_{t}\right), \hat{X}_{t}^{\theta}\right\rangle d \lambda d t \\
& \leq \limsup _{T \rightarrow \infty} \frac{1}{T} \int_{0}^{T} \int_{0}^{1}\left(\mathbb{E}\left|D_{x} f\left(\bar{X}_{t}+\lambda\left(X_{t}^{\theta}-\bar{X}_{t}\right), \bar{u}_{t}+\lambda \theta v_{t}\right)\right|^{2}\right)^{1 / 2}\left(\mathbb{E}\left|\hat{X}_{t}^{\theta}\right|^{2}\right)^{1 / 2} d \lambda d t
\end{aligned}
$$

which converges to zero, uniformly in $T$, as $\theta \rightarrow 0_{+}$. In fact, this follows from the linear growth of $D_{x} f(\cdot)$, the a priori estimates on $X_{t}$ and Lemma 1 The conclusion now easily follows.

\section{The ADJOINT Equation}

In this section we introduce the dual equation associated to the system, which is an infinite horizon Backward SDE in $\mathbb{R}^{n}$. Different approaches have been developed in the literature to study this class of equations. Here we present a duality method built on construction of a family of truncated equations and associated with a consistency argument. More precisely, the infinite horizon backward equation has the form

$$
-d p_{t}=\left[D_{x} b\left(X_{t}, u_{t}\right)^{*} p_{t}+\sum_{i=1}^{d} D_{x} \sigma^{i}\left(X_{t}, u_{t}\right)^{*} q_{t}^{i}-D_{x} f\left(X_{t}, u_{t}\right)\right] d t-\sum_{i=1}^{d} q_{t}^{i} d W_{t}^{i}
$$

where, fixed any orthonormal basis $\left(e_{i}\right)_{i=1, . . d}$ in $\mathbb{R}^{d}$ we set $W_{s}^{i}=<e_{i}, W_{s}>$ and $\sigma^{i}(x, u)=\sigma(x, u) e_{i}$ moreover we denote by $(\cdot)^{*}$ the transposition operation in $\mathcal{L}\left(\mathbb{R}^{n}\right)$. For every $T>0$ fixed, its solution has to be understood as

$$
p_{t}=p_{T}+\int_{t}^{T}\left[D_{x} b\left(X_{s}, u_{s}\right)^{*} p_{s}+\sum_{i=1}^{d} D_{x} \sigma^{i}\left(X_{s}, u_{s}\right)^{*} q_{s}^{i}+D_{x} f\left(X_{s}, u_{s}\right)\right] d s-\sum_{i=1}^{d} \int_{t}^{T} q_{s}^{i} d W_{s}^{i} .
$$

where $p$ and $q^{i}, i=1, \ldots, d$ take values in $\mathbb{R}^{n}$. Due to Hypothesis 1 and estimate (14) the forcing term in the driver is no better than bounded, so that $D_{x} f\left(X_{s}, u_{s}\right) \in L^{\infty}\left(\mathbb{R}_{+} ; L^{2}\left(\Omega ; \mathbb{R}^{n}\right)\right)$. Therefore we cannot expect the solution of (34) to be integrable up to infinity but only that $p \in L^{\infty}\left(\mathbb{R}_{+} ; L^{2}\left(\Omega ; \mathbb{R}^{n}\right)\right)$. Up to the authors17 knowledge, there is not a general wellposendess result for such multidimensional BSDE's. Partial results have been obtained in [5] by a Girsanov argument that seems to work only if one knows a-priori that $\sum_{i=1}^{d} D_{x} \sigma^{i}\left(X_{s}, u_{s}\right)^{*} q_{s}^{i}$ can be written as $\sum_{i=1}^{d} q_{s}^{i} f^{i}$ for suitable adapted real process $\left(f_{i}\right)_{i=1, \ldots, d}$. In particular this is the case when $n=1$ or the noise is additive.

Here the solution will be obtained via the introduction of a family of time truncations:

$$
\left\{\begin{array}{l}
-d p_{t}^{T, \nu}=\left[D_{x} b\left(X_{t}, u_{t}\right)^{*} p_{t}^{T, \nu}+\sum_{i=1}^{d} D_{x} \sigma^{i}\left(X_{t}, u_{t}\right)^{*} q_{t}^{i, T, \nu}-D_{x} f\left(X_{t}, u_{t}\right)\right] d t-\sum_{i=1}^{d} q_{t}^{i, T, \nu} d W_{t}^{i}, \\
p_{T}^{T, \nu}=\nu .
\end{array}\right.
$$

which will be estimated by duality. For the approximating equation (36) a wellposedness result has been already adressed in [15].

To shorten the notation in the following paragraphs, let us denote

$$
\Lambda_{t}:=D_{x} b\left(X_{t}, u_{t}\right), \quad \Gamma_{t}^{i}:=D_{x} \sigma^{i}\left(X_{t}, u_{t}\right), \quad \Psi_{t}:=D_{x} f\left(X_{t}, u_{t}\right)
$$

moreover when $\nu=0$ the solution of equation (36) will be denoted by $\left(p^{T}, q^{i, T}\right)$.

Theorem 2. For all $T \geq 0$ and all $\nu \in L^{2}\left(\Omega, \mathcal{F}_{T}, \mathbb{P} ; \mathbb{R}^{n}\right)$ there exists a unique $(d+1)$-tuple of $\mathbb{R}^{n}$-valued, adapted processes $\left(p^{T, \nu}, q^{1, T, \nu}, \ldots, q^{d, T, \nu}\right)$ such that $p^{T, \nu}$ has continuous trajectories, $\sup _{t \in[0, T]} \mathbb{E}\left|p_{t}^{T, \nu}\right|^{2}+\sum_{i=1}^{d} \mathbb{E} \int_{0}^{T}\left|q_{t}^{i, T, \nu}\right|^{2} d t<\infty$ and, $\mathbb{P}$-almost surely, for all $t \in[0, T]$ it holds:

$$
p_{t}^{T, \nu}=\nu+\int_{t}^{T} \Lambda_{s}^{*} p_{s}^{T, \nu} d s+\sum_{i=1}^{d} \int_{t}^{T}\left(\Gamma_{s}^{i}\right)^{*} q^{i, T, \nu} d s+\int_{t}^{T} \Psi_{s} d s+\sum_{i=1}^{d} \int_{t}^{T} q_{s}^{i, T, \nu} d W_{s}^{i}
$$


Consider now the following affine forward SDE with general forcing term $\left(\gamma, \rho^{i}\right)_{i=1, . .,}$ with $\gamma$ and $\rho^{i}, i=1, . ., d$ in $L^{2}\left([0, T] ; L^{2}\left(\Omega ; \mathbb{R}^{n}\right)\right)$ and initial condition $\eta \in L^{2}\left(\Omega, \mathcal{F}_{t} ; \mathbb{R}^{n}\right)$ :

$$
\left\{\begin{array}{l}
d \mathcal{Y}_{s}^{t, \eta, \gamma, \rho}=\Lambda_{s} \mathcal{Y}_{s}^{t, \eta, \gamma, \rho} d t+\sum_{i=1}^{d} \Gamma_{t}^{i} \mathcal{Y}_{s}^{t, \eta, \gamma, \rho} d W_{t}^{i}+\gamma_{s} d s+\sum_{i=1}^{d} \rho_{s}^{i} d W_{s}^{i}, s \geq t, \\
\mathcal{Y}_{t}^{t, \eta, \gamma, \rho}=\eta
\end{array}\right.
$$

Then by the same technique we adopted in the proof of Theorem 1, the above equation admits a unique adapted solution and

$$
\mathbb{E}\left|\mathcal{Y}_{r}^{t, \eta, \gamma, \rho}\right|^{2} \leq e^{-2 \beta(r-t)} \mathbb{E}|\eta|^{2}+K \int_{t}^{r} e^{-2 \beta(r-s)} \mathbb{E}\left[\left|\gamma_{s}\right|^{2}+\left|\rho_{s}^{1}\right|^{2}+\ldots+\left|\rho_{s}^{d}\right|^{2}\right] d s .
$$

When $\gamma \equiv 0$ then the solution to the above equation will be denoted by $\mathcal{Y}^{t, \eta, \rho}$ and when $\rho \equiv 0$ as well, it will be denoted by $\mathcal{Y}^{t, \eta, \gamma}$.

The next result is proven in [15] by computing the Itô formula the differential of the product $d\left\langle Y_{s}^{t, \eta, \gamma, \rho}, p_{s}^{T, \nu}\right\rangle$

Lemma 4. Given $\left(\rho^{i}\right)_{i=1, . ., d}$ with $\gamma, \rho^{i} \in L^{2}\left([0, T] ; L^{2}\left(\Omega ; \mathbb{R}^{n}\right)\right), \eta \in L^{2}\left(\Omega, \mathcal{F}_{t} ; \mathbb{R}^{n}\right), \nu \in L^{2}\left(\Omega, \mathcal{F}_{T} ; \mathbb{R}^{n}\right)$ it holds:

$$
\mathbb{E} \int_{t}^{T}\left\langle p_{s}^{T, \nu}, \gamma_{s}\right\rangle d s+\sum_{i=1}^{d} \mathbb{E} \int_{t}^{T}\left\langle q_{s}^{i, T, \nu}, \rho_{s}^{i}\right\rangle d s+\mathbb{E}\left\langle p_{t}^{T, \nu}, \eta\right\rangle=\mathbb{E} \int_{t}^{T}\left\langle\mathcal{Y}_{s}^{t, \eta, \gamma, \rho}, \Psi_{s}\right\rangle d s+\mathbb{E}\left\langle\nu, \mathcal{Y}_{T}^{t, \eta, \gamma, \rho}\right\rangle .
$$

In the following, relation (39) will be the main instrument to get information on the behaviour of the BSDE. We will specifically choose the values of $t, \eta, \rho$ according to our needs.

We are now in a position to define the solution to the infinite horizon multidimensional BSDE and prove its existence and uniqueness

Definition 1. A solution to equation (34) is a $(d+1)$-tuple of $\mathbb{R}^{n}$-valued, adapted processes $\left(p_{t}, q_{t}^{1}, \ldots, q_{t}^{d}\right)_{t \in[0, \infty[}$ such that, for all $T>0$ and all $i=1, \ldots, d$ it holds $\mathbb{E} \int_{0}^{T}\left|q_{t}^{i}\right|^{2} d t<\infty$. Moreover $p$ has continuous trajectories and $\sup _{t \in[0, \infty)} \mathbb{E}\left|p_{t}\right|^{2}<\infty$. Finally, for all $0 \leq t \leq T$, (35) holds $\mathbb{P}$-almost surely.

The main result of this section is the following

Theorem 3. Let Hypothesis 1 holds true. Then equation (34) admits a unique solution ( $p^{\infty}$, $\left.q^{1, \infty}, \ldots, q^{d, \infty}\right)$.

Proof.

Existence: Let in (39) $\nu \equiv 0, \gamma \equiv 0, \rho \equiv 0, \eta \in L^{2}\left(\Omega, \mathcal{F}_{t} ; \mathbb{R}^{n}\right)$ then

$$
\mathbb{E}\left\langle p_{t}^{T}, \eta\right\rangle=\mathbb{E} \int_{t}^{T}\left\langle\mathcal{Y}_{s}^{t, \eta}, \Psi_{s}\right\rangle d s .
$$

Since $\Psi \in L^{\infty}\left(\mathbb{R}_{+} ; L^{2}\left(\Omega ; \mathbb{R}^{n}\right)\right)$ by (38) we deduce that

$$
\mathbb{E} \int_{t}^{T}\left\langle\mathcal{Y}_{s}^{t, \eta}, \Psi_{s}\right\rangle d s \rightarrow \mathbb{E} \int_{t}^{\infty}\left\langle\mathcal{Y}_{s}^{t, \eta}, \Psi_{s}\right\rangle d s
$$

and that the right hand side is a bounded linear operator from $L^{2}\left(\Omega, \mathcal{F}_{t} ; \mathbb{R}^{n}\right) \rightarrow \mathbb{R}$. Hence, by Riesz representation theorem there exists an element $P(t) \in L^{2}\left(\Omega, \mathcal{F}_{t} ; \mathbb{R}^{n}\right)$ such that

$$
\mathbb{E}\langle P(t), \eta\rangle=\mathbb{E} \int_{t}^{\infty}\left\langle\mathcal{Y}_{s}^{t, \eta}, \Psi_{s}\right\rangle d s .
$$

Moreover $p^{T}(t) \rightarrow P(t)$ in $L^{2}\left(\Omega, \mathcal{F}_{t}, \mathbb{P} ; \mathbb{R}^{n}\right)$ and $\mathbb{E}|P(t)|^{2} \leq \beta^{-1} \sup _{s \in[0, \infty[}\left(\mathbb{E}\left|\Psi_{s}\right|^{2}\right)^{1 / 2}$ for all $t>0$.

Let now for all $N \in \mathbb{N},\left(\tilde{p}_{t}^{N}, \tilde{q}_{t}^{1, N}, \ldots, \tilde{q}_{t}^{d, N}\right)_{t \in[0, N]}$ be the solution of equation (36) with $T=N$ and $\nu=P(N)$.

We claim that, for all $N, M \in \mathbb{N}$ with $0 \leq N \leq M$ and all $t \leq N$ it holds

$$
\tilde{p}^{N}(t)=\tilde{p}^{M}(t), \mathbb{P} \text {-a.s. }
$$

By definition and Lemma 4 we deduce that for all $\eta \in L^{2}\left(\Omega, \mathcal{F}_{t}, \mathbb{P} ; \mathbb{R}^{n}\right)$

$$
\mathbb{E}\left\langle\tilde{p}_{t}^{N}, \eta\right\rangle=\mathbb{E} \int_{t}^{N}\left\langle\mathcal{Y}_{s}^{t, \eta}, \Psi_{s}\right\rangle d s+\mathbb{E}\left\langle P(N), \mathcal{Y}_{N}^{t, \eta}\right\rangle .
$$


Plugging (41) with $t=N$ in the above relation we have

$$
\mathbb{E}\left\langle\tilde{p}_{t}^{N}, \eta\right\rangle=\mathbb{E} \int_{t}^{N}\left\langle\mathcal{Y}_{s}^{t, \eta}, \Psi_{s}\right\rangle d s+\mathbb{E} \int_{N}^{\infty}\left\langle\mathcal{Y}_{s}^{N, \mathcal{Y}_{N}^{t, \eta}}, \Psi_{s}\right\rangle d s
$$

and finally, observing that by uniqueness of the solution to equation (37) $\mathcal{Y}_{s}^{N, \mathcal{Y}_{N}^{t, \eta}}=\mathcal{Y}_{s}^{t, \eta} \mathbb{P}$-a.s. we conclude

$$
\mathbb{E}\left\langle\tilde{p}_{t}^{N}, \eta\right\rangle=\mathbb{E} \int_{t}^{\infty}\left\langle\mathcal{Y}_{s}^{t, \eta}, \Psi_{s}\right\rangle d s=\mathbb{E}\langle\eta, P(t)\rangle .
$$

and our claim is proved since the right hand side does not depend on $N$. We also remark that by the above identity we deduce that

$$
\sup _{t \in[0, N]}\left|\tilde{p}_{t}^{N}\right|^{2} \leq \beta^{-1} \sup _{s \in[0, \infty[}\left(\mathbb{E}\left|\psi_{s}\right|^{2}\right)^{1 / 2},
$$

and that the right hand side does not depend neither on $t$ nor on $N$.

Now we define

$$
p_{t}^{\infty}=\sum_{N=1}^{\infty} \tilde{p}_{t}^{N} I_{[N-1, N[}(t), \quad q_{t}^{i, \infty}=\sum_{N=1}^{\infty} \tilde{q}_{t}^{i, N} I_{[N-1, N[}(t),
$$

and claim that it is the desired solution. Indeed it satisfies the desired integrability and adaptedness conditions. Moreover fixed $0 \leq t \leq T$ then

$$
\begin{aligned}
p_{t}^{\infty}-p_{T}^{\infty} & =\left[p_{t}^{\infty}-p_{\lfloor t\rfloor+1}^{\infty}\right]+\left[p_{\lfloor T\rfloor}^{\infty}-p_{T}^{\infty}\right]+\sum_{n=\lfloor t\rfloor+1}^{\lfloor T\rfloor-1}\left[p_{n}^{\infty}-p_{n+1}^{\infty}\right] \\
& =\left[\tilde{p}_{t}^{\lfloor t\rfloor+1}-\tilde{p}_{\lfloor t\rfloor+1}^{\lfloor t\rfloor+2}\right]+\left[\tilde{p}_{\lfloor T\rfloor}^{\lfloor T\rfloor+1}-\tilde{p}_{T}^{\lfloor T\rfloor+1}\right]+\sum_{n=\lfloor t\rfloor+1}^{\lfloor T\rfloor-1}\left[\tilde{p}_{n}^{n+1}-\tilde{p}_{n+1}^{n+2}\right] \\
& =\left[\tilde{p}_{t}^{\lfloor t\rfloor+1}-\tilde{p}_{\lfloor t\rfloor+1}^{\lfloor t\rfloor+1}\right]+\left[\tilde{p}_{\lfloor T\rfloor}^{\lfloor T\rfloor+1}-\tilde{p}_{T}^{\lfloor T\rfloor+1}\right]+\sum_{n=\lfloor t\rfloor+1}^{\lfloor T\rfloor-1}\left[\tilde{p}_{n}^{n+1}-\tilde{p}_{n+1}^{n+1}\right],
\end{aligned}
$$

where in the last equality we have exploited (42) where it was needed. Now recalling that $\left(\tilde{p}_{t}^{N}, \tilde{q}_{t}^{1, N}, \ldots, \tilde{q}_{t}^{d, N}\right)_{t \in[0, N]}$ solves equation (36) and the definition of $\left(p^{\infty}, q^{1, \infty}, \ldots, \tilde{q}^{d, \infty}\right)$ the above equality can be rewritten as

$$
\begin{aligned}
p_{t}^{\infty}-p_{T}^{\infty}= & \int_{t}^{\lfloor t\rfloor+1} \Lambda_{s}^{*} p_{s}^{\infty} d s+\sum_{i=1}^{d} \int_{t}^{\lfloor t\rfloor+1}\left(\Gamma_{s}^{i}\right)^{*} q_{s}^{i, \infty} d s+\int_{t}^{\lfloor t\rfloor+1} \Psi_{s} d s+\sum_{i=1}^{d} \int_{t}^{\lfloor t\rfloor+1} q_{s}^{i, \infty} d W_{s}^{i} \\
& +\sum_{n=\lfloor t\rfloor+1}^{\lfloor T\rfloor-1}\left[\int_{n}^{n+1} \Lambda_{s}^{*} p_{s}^{\infty} d s+\sum_{i=1}^{d} \int_{n}^{n+1}\left(\Gamma_{s}^{i}\right)^{*} q_{s}^{i, \infty} d s+\int_{n}^{n+1} \Psi_{s} d s+\sum_{i=1}^{d} \int_{n}^{n+1} q_{s}^{i, \infty} d W_{s}^{i}\right] \\
& +\int_{\lfloor T\rfloor}^{T} \Lambda_{s}^{*} p_{s}^{\infty} d s+\sum_{i=1}^{d} \int_{\lfloor T\rfloor}^{T}\left(\Gamma_{s}^{i}\right)^{*} q_{s}^{i, \infty} d s+\int_{\lfloor T\rfloor}^{T} \Psi_{s} d s+\sum_{i=1}^{d} \int_{\lfloor T\rfloor}^{T} q_{s}^{i, \infty} d W_{s}^{i} \\
= & \int_{t}^{T} \Lambda_{s}^{*} p_{s}^{\infty} d s+\sum_{i=1}^{d} \int_{t}^{T}\left(\Gamma^{i}\right)_{s}^{*} q_{s}^{i, \infty} d s+\int_{t}^{T} \Psi_{s} d s+\sum_{i=1}^{d} \int_{t}^{T} q_{s}^{i, \infty} d W_{s}^{i}
\end{aligned}
$$

and this completes the proof of existence of a solution to equation (35).

Uniqueness: Let $\left(p_{t}, q_{t}^{1}, \ldots, q_{t}^{d}\right)_{t \geq 0}$ be a solution to equation (35). We choose $\rho \in L^{2}\left(\Omega \times\left[0, \infty\left[; \mathbb{R}^{n}\right)\right.\right.$ with support in the finite interval $[0, T]\left(\rho_{r}=0\right.$, if $\left.r \geq T\right)$ and $\eta \in L^{2}\left(\Omega, \mathcal{F}_{t}, \mathbb{P} ; \mathbb{R}^{n}\right)$. Noticing that $\left(p_{t}, q_{t}^{1}, \ldots, q_{t}^{d}\right)_{t \geq 0}$ is, in particular a solution to equation (36) in $[0, T]$ with $\nu=p_{T}$ by Lemma 4 we get:

$$
\mathbb{E} \int_{t}^{T}\left\langle\mathcal{Y}_{s}^{t, \eta, \rho}, \Psi_{s}\right\rangle d s+\mathbb{E}\left\langle p_{T}, \mathcal{Y}_{T}^{t, \eta, \rho}\right\rangle=\sum_{i=1}^{d} \mathbb{E} \int_{t}^{T}\left\langle q_{s}^{i}, \rho_{s}\right\rangle d s+\mathbb{E}\left\langle\eta, \tilde{p}_{t}\right\rangle .
$$


We notice that since $\rho_{t}=0$ for $t>T$ then by (38) we have that $\mathbb{E}\left|\mathcal{Y}_{s}^{t, \eta, \rho}\right|^{2} \leq C e^{-2 \beta(s-t)}$ for a suitable $C$. So letting $T \rightarrow \infty$ in the above equality we get (recall that $\sup _{t \geq 0} \mathbb{E}\left|p_{t}\right|^{2}<\infty$ by definition of solution):

$$
\mathbb{E} \int_{t}^{\infty}\left\langle\mathcal{Y}_{s}^{t, \eta, \rho}, \Psi_{s}\right\rangle d s=\sum_{i=1}^{d} \mathbb{E} \int_{t}^{T}\left\langle q_{s}^{i}, \rho_{s}\right\rangle d s+\mathbb{E}\left\langle\eta, \tilde{p}_{t}\right\rangle
$$

and this completes the proof of uniqueness due to the arbitrariness of $t, T, \rho$ and $\eta$.

As a by-product of the above proof we have the following infinite-horizon version of the duality relation:

Corollary 4. Let $\left(p_{t}, q_{t}^{1}, \ldots, q_{t}^{d}\right)_{t \geq 0}$ be a solution to equation (35). Fix $\rho \in L^{2}\left(\Omega \times\left[0, \infty\left[; \mathbb{R}^{n}\right)\right.\right.$ with support in $[0, T], t \in[0, T)$ and $\eta \in L^{2}\left(\Omega, \mathcal{F}_{t}, \mathbb{P} ; \mathbb{R}^{n}\right)$ then (45) holds.

\section{NeCEssary ERgodic SMP}

We give two versions of the SMP in its necessary form. The first is based on the well-posedness result for the infinite horizon BSPDE. The second one is written in terms of the family of truncated backward equations introduced in the previous section. The Hamiltonian associated to the system is

$$
H\left(x, u, p, q^{1}, \ldots q^{d}\right)=\langle b(x, u), p\rangle+\sum_{i=1}^{d}\left\langle\sigma^{i}(x, u), q^{i}\right\rangle+f(x, u) .
$$

We are now in a condition to formulate a necessary condition corresponding to the ergodic control problem.

Theorem 5 (SMP infinite horizon case). Suppose that $(\bar{X}, \bar{u})$ is an optimal pair for the control problem $J^{\inf }$ or $J^{\text {sup }}$ and let $\left(p^{\infty}, q^{\infty}\right)=\left(p^{\infty}, q^{\infty, 1}, \ldots, q^{\infty, d}\right)$ be the solution of equation (34). Then under Hypothesis 1, the following variational inequality holds:

$$
0 \leq \limsup _{T \rightarrow \infty} \frac{1}{T} \mathbb{E} \int_{0}^{T}\left\langle D_{u} H\left(\bar{X}_{t}, \bar{u}_{t}, p_{t}^{\infty}, q_{t}^{\infty}\right), u_{t}-\bar{u}_{t}\right\rangle_{\mathbb{R}^{l}} d t
$$

where $H(x, u, p, q)$ is the Hamiltonian of the system, and $u(\cdot)$ is an arbitrary admissible control.

Proof. Let $v(\cdot)=u(\cdot)-\bar{u}(\cdot)$ and let $Y_{t}$ be the solution to equation (21). Lemma 4 with $t=0$, $\eta=0, \nu=p_{T}^{\infty}, \gamma=D_{u} b(\bar{X}, \bar{u}), \rho^{i}=D_{u} \sigma(\bar{X}, \bar{u}) v$ yields

$$
\begin{aligned}
& \mathbb{E} \int_{0}^{T}\left\langle D_{x} f\left(\bar{X}_{t}, \bar{u}_{t}\right), Y_{t}\right\rangle d t \\
& =\mathbb{E}\left\langle p_{T}^{\infty}, Y_{T}\right\rangle+\mathbb{E} \int_{0}^{T}\left\langle p_{t}^{\infty}, D_{u} b\left(\bar{X}_{t}, \bar{u}_{t}\right) v_{t}\right\rangle d t+\mathbb{E} \int_{0}^{T}\left\langle q_{t}^{\infty}, D_{u} \sigma\left(\bar{X}_{t}, \bar{u}_{t}\right) v_{t}\right\rangle d t .
\end{aligned}
$$

So that, from Lemma 3 and the relation above, we have

$$
\begin{aligned}
0 & \leq \limsup _{T \rightarrow \infty} \frac{1}{T} \mathbb{E} \int_{0}^{T}\left[\left\langle D_{x} f\left(\bar{X}_{t}, \bar{u}_{t}\right), Y_{t}\right\rangle_{\mathbb{R}^{n}}+\left\langle D_{u} f\left(\bar{X}_{t}, \bar{u}_{t}\right), v_{t}\right\rangle_{\mathbb{R}^{l}}\right] d t . \\
& \leq-\limsup _{T \rightarrow \infty} \frac{1}{T} \mathbb{E}\left\langle Y_{T}, p_{T}^{\infty}\right\rangle+\limsup _{T \rightarrow \infty} \frac{1}{T} \mathbb{E} \int_{0}^{T}\left[\left\langle D_{u} H\left(\bar{X}_{t}, \bar{u}_{t}, p_{t}^{\infty}, q_{t}^{\infty}\right), v_{t}\right\rangle_{\mathbb{R}^{l}}\right] d t .
\end{aligned}
$$

Recalling that $\sup _{t \geq 0} \mathbb{E}\left|p_{t}^{\infty}\right|^{2}<+\infty$ by definition of solution to equation (34) and $\sup _{t \geq 0} \mathbb{E}\left|Y_{t}\right|^{2}<$ $+\infty$ by (38) we can conclude that

$$
0 \leq \limsup _{T \rightarrow \infty} \frac{1}{T} \mathbb{E} \int_{0}^{T}\left[\left\langle D_{u} H\left(\bar{X}_{t}, \bar{u}_{t}, p_{t}^{\infty}, q_{t}^{\infty}\right), v_{t}\right\rangle_{\mathbb{R}^{l}}\right] d t
$$

and the claim is proved

Remark 4 (SMP truncated case). Similarly we can prove a truncated version of the stochastic maximum principle that involves the solution $\left(p^{T}, q^{1, T}, \ldots q^{d, T}\right)$ of equation (36) with $\eta=0$. Indeed 
if $(\bar{X}, \bar{u})$ is an optimal pair for the control problem (9), then under Hypothesis 1 the following variational inequality holds

$$
0 \leq \limsup _{T \rightarrow \infty} \frac{1}{T} \mathbb{E} \int_{0}^{T}\left\langle D_{u} H\left(\bar{X}_{t}, \bar{u}_{t}, p_{t}^{T}, q_{t}^{T}\right), u_{t}-\bar{u}_{t}\right\rangle_{\mathbb{R}^{l}} d t,
$$

where $H(x, u, p, q)$ is the Hamiltonian of the system and $u(\cdot)$ is an arbitrary admissible control.

Proof. Let $v_{t}=u_{t}-\bar{u}_{t}$, for every $u_{t}$ admissible. The result easily follows combining Lemma 3 with a duality argument. Precisely, choose $\eta=0, \nu_{t}=D_{u} b\left(\bar{X}_{t}, \bar{u}_{t}\right) v_{t}$ and $\Psi_{t}=D_{x} f\left(\bar{X}_{t}, \bar{u}_{t}\right)$ in the general formula (37).

\section{Sufficient SMP}

In this part we prove that under some additional convexity assumption on the Hamiltonian function $H$, the variational inequality obtained in Theorem 5 (the same hold also for Theorem 4) is sufficient for optimality.

Theorem 6 (Sufficient SMP). Let $u^{*}(\cdot) \in \mathcal{U}_{a d}$ be an admissible control, $X^{*}$ be the corresponding state process and $p^{*}$ the first adjoint process on infinite time horizon solving (34) for the couple $\left(u^{*}, X^{*}\right)$. Further, let $(x, u) \mapsto H\left(x, u, p_{t}^{*}, q_{t}^{*}\right)$ be a convex function $d \mathbb{P} \times d t-a . e$. and the following minimality condition holds

$$
\limsup _{T \rightarrow+\infty} \frac{1}{T} \mathbb{E} \int_{0}^{T}\left\langle D_{u} H\left(X_{t}^{*}, u_{t}^{*}, p_{t}^{*}, q_{t}^{*}\right), u_{t}-u_{t}^{*}\right\rangle_{\mathbb{R}^{l}} d t \geq 0,
$$

for every $u(\cdot) \in \mathcal{U}_{\text {ad }}$. Then $u^{*}(\cdot)$ is optimal both for lim inf and lim sup formulations of the ergodic control problem.

Proof. Let $u(\cdot) \in \mathcal{U}_{a d}$ be arbitrary but fixed. Then the goal is to show that the difference $J\left(u^{*}(\cdot)\right)-$ $J(u(\cdot))$ is non-positive. Using the sub additivity of the limsup we have

$$
\begin{aligned}
J\left(u^{*}(\cdot)\right)- & J(u(\cdot)) \leq \limsup _{T \rightarrow+\infty} \frac{1}{T} \mathbb{E} \int_{0}^{T}\left[f\left(X_{t}^{*}, u_{t}^{*}\right)-f\left(X_{t}, u_{t}\right)\right] d t \\
& =\limsup _{T \rightarrow+\infty} \frac{1}{T} \mathbb{E} \int_{0}^{T}\left[H\left(X_{t}^{*}, u_{t}^{*}, p_{t}^{*}, q_{t}^{*}\right)-H\left(X_{t}, u_{t}, p_{t}^{*}, q_{t}^{*}\right)\right] d t \\
& +\limsup _{T \rightarrow+\infty} \frac{1}{T} \mathbb{E} \int_{0}^{T}\left\langle b\left(X_{t}, u_{t}\right)-b\left(X_{t}^{*}, u_{t}^{*}\right), p_{t}^{*}\right\rangle d t \\
& +\limsup _{T \rightarrow+\infty} \frac{1}{T} \mathbb{E} \int_{0}^{T}\left\langle\sigma\left(X_{t}, u_{t}\right)-\sigma\left(X_{t}^{*}, u_{t}^{*}\right), q_{t}^{*}\right\rangle d t=I_{1}+I_{2}+I_{3} .
\end{aligned}
$$

Now, due to convexity of $H$, the term $I_{1}$ can be estimated from above as follows

$$
\begin{aligned}
I_{1} \leq & \limsup _{T \rightarrow+\infty} \frac{1}{T} \mathbb{E} \int_{0}^{T}\left\langle D_{x} H\left(X_{t}^{*}, u_{t}^{*}, p_{t}^{*}, q_{t}^{*}\right), X_{t}^{*}-X_{t}\right\rangle d t \\
& +\limsup _{T \rightarrow+\infty} \frac{1}{T} \mathbb{E} \int_{0}^{T}\left\langle D_{u} H\left(X_{t}^{*}, u_{t}^{*}, p_{t}^{*}, q_{t}^{*}\right), u_{t}^{*}-u_{t}\right\rangle_{U} d t \\
\leq & \limsup _{T \rightarrow+\infty} \frac{1}{T} \mathbb{E} \int_{0}^{T}\left\langle D_{x} H\left(X_{t}^{*}, u_{t}^{*}, p_{t}^{*}, q_{t}^{*}\right), X_{t}^{*}-X_{t}\right\rangle d t
\end{aligned}
$$

where in the last step we have used the minimality condition (50). Next,

$$
\lim _{T \rightarrow \infty} \frac{1}{T} \mathbb{E}\left\langle p_{T}^{*}, X_{T}^{*}-X_{T}\right\rangle=0,
$$

due to the fact that $p^{*}, X, X^{*} \in L^{\infty}\left(\mathbb{R}_{+} ; L^{2}(\Omega ; H)\right)$.

By applying the Itô formula to $\left\langle p_{T}^{*, T}, X_{T}^{*}-X_{T}\right\rangle_{H}$ and putting all the terms together we arrive at

$$
J(u(\cdot))-J\left(u^{*}(\cdot)\right) \leq 0
$$

The above inequality means that $u^{*}(\cdot)$ is optimal control. 
The form of minimality condition (50) is related to our definition of the Hamiltonian. In fact, one could introduce an another sign convention for $H$, namely $H(x, u, p, q)=\langle b(t, x, u), p\rangle+$ $\sum_{i=1}^{d}\left\langle\sigma^{i}(x, u), q^{i}\right\rangle-f(x, u)$ which would lead to the corresponding modification in the driver of the first adjoint equation, concavity assumption (instead of convexity) on $H$ in $(x, u)$ and the opposite inequality in (50). All these changes would lead to the maximality condition usually considered with stochastic maximum principle.

\section{REFERENCES}

[1] M. Arisawa and P.-L. Lions. On ergodic stochastic control. Comm. Partial Differential Equations, 23(11-12):2187-2217, 1998.

[2] A. Bensoussan. Équations paraboliques intervenant en contrôle optimal ergodique. Mat. Apl. Comput., 6(3):211-255, 1987.

[3] Vivek S Borkar and Mrinal K Ghosh. Ergodic control of multidimensional diffusions. I. The existence results. SIAM Journal on Control and Optimization, 26(1):112-126, 1988.

[4] Sandra Cerrai. Second order PDE's in finite and infinite dimension, volume 1762 of Lecture Notes in Mathematics. Springer-Verlag, Berlin, 2001. A probabilistic approach.

[5] Samuel N Cohen and Victor Fedyashov. Classical adjoints for ergodic stochastic control. arXiv preprint arXiv:1511.04255, 2015.

[6] Arnaud Debussche, Ying Hu, and Gianmario Tessitore. Ergodic BSDEs under weak dissipative assumptions. Stochastic Process. Appl., 121(3):407-426, 2011.

[7] Marco Fuhrman, Ying Hu, and Gianmario Tessitore. Ergodic BSDES and optimal ergodic control in Banach spaces. SIAM J. Control Optim., 48(3):1542-1566, 2009.

[8] Beniamin Goldys and Bohdan Maslowski. Ergodic control of semilinear stochastic equations and the Hamilton-Jacobi equation. J. Math. Anal. Appl., 234(2):592-631, 1999.

[9] Beniamin Goldys and Bohdan Maslowski. On stochastic ergodic control in infinite dimensions. In Seminar on Stochastic Analysis, Random Fields and Applications VI, volume 63 of Progr. Probab., pages 95-107. Birkhäuser/Springer Basel AG, Basel, 2011.

[10] Giuseppina Guatteri and Federica Masiero. Ergodic optimal quadratic control for an affine equation with stochastic and stationary coefficients. Systems Control Lett., 58(3):169-177, 2009.

[11] Ying $\mathrm{Hu}$, Pierre-Yves Madec, and Adrien Richou. A probabilistic approach to large time behavior of mild solutions of HJB equations in infinite dimension. SIAM J. Control Optim., 53(1):378-398, 2015.

[12] H. J. Kushner. Optimality conditions for the average cost per unit time problem with a diffusion model. SIAM J. Control Optimization, 16(2):330-346, 1978.

[13] Petr Mandl. On control by non-stopped diffusion processes. Teor. Verojatnost. i Primenen., 9:655-669, 1964.

[14] Bohdan Maslowski and Petr Veverka. Sufficient stochastic maximum principle for discounted control problem. Appl. Math. Optim., 70(2):225-252, 2014.

[15] Carlo Orrieri and Petr Veverka. Necessary stochastic maximum principle for dissipative systems on infinite time horizon. ESAIM: Control Optim. Calc. Var., to appear.

[16] Shige Peng. A general stochastic maximum principle for optimal control problems. SIAM J. Control Optim., 28(4):966-979, 1990.

[17] Adrien Richou. Ergodic BSDEs and related PDEs with Neumann boundary conditions. Stochastic Process. Appl., 119(9):2945-2969, 2009.

(C. Orrieri) Dipartimento di Matematica, Sapienza Università di Roma. Piazzale Aldo Moro 5, 00185 Roma, ITALia

E-mail address: orrieri@mat.uniroma1.it

(G. Tessitore) Dipartimento di Matematica e Applicazioni, Universitì di Milano-Bicocca. Via Cozzi 55, 20125 Milano, Italia

E-mail address: gianmario.tessitore@unimib.it

(P. Veverka) Institute of Information Theory and Automation, Czech Academy of Sciences, Pod Vodárenskou věží 4, Praha 8, 182 08, Czech Republic

E-mail address: panveverka@seznam.cz 